\title{
Colorectal cancer pain upon diagnosis and after treatment: a cross-sectional comparison with healthy matched controls
}

\author{
Maria Lopez-Garzon ${ }^{1,2,3} \cdot$ Paula Postigo-Martin 1,2,3 (10 - Ángela González-Santos 1,2,3 Manuel Arroyo-Morales $^{1,2,3,4}$. \\ Alexander Achalandabaso-Ochoa ${ }^{3}$. Antonio Manuel Férnández-Pérez ${ }^{2} \cdot$ Irene Cantarero-Villanueva ${ }^{1,2,3,4}$
}

Received: 13 August 2021 / Accepted: 31 December 2021 / Published online: 13 January 2022

(c) The Author(s) 2022

\begin{abstract}
Background The current study sought to explore whether cancer pain (CP) already exists in patients at colorectal cancer (CRC) diagnosis before treatment compared with patients with colorectal cancer (CRC) after treatment and a healthy matched control group. The study also sought to examine whether factors related to physical health status could enhance pain processes.

Methods An observational cross-sectional study was conducted following the STROBE checklist. Twenty-nine newly diagnosed and forty post-treatment patients with CRC and 40 healthy age/sex-matched controls were included for comparison. Pain, local muscle function, and body composition outcomes were assessed by a physiotherapist with $>3$ years of experience. ANCOVA and Kruskal-Wallis tests were performed, with Bonferroni and Dunn-Bonferroni post hoc analyses and Cohen's $d$ and Hedge's effect size, as appropriate.

Results The analysis detected lower values of pressure pain threshold (PPT) points, the PPT index, and abdominal strength and higher values of self-reported abdominal pain in newly diagnosed patients, with even more marked results observed in the post-treatment patients, where lower lean mass and skeletal muscle index values were also found than those in the healthy matched controls $(p<0.05)$. In the post-treatment and healthy matched control groups, positive associations were observed between the PPT lumbar dominant side points and abdominal isometric strength and lean mass, and negative associations were observed between the lumbar dominant side points and body fat $(p<0.05)$.

Conclusion Upon diagnosis, patients with CRC already show signs of hyperalgesia and central sensitization and deteriorated physical conditions and body composition, and this state could be aggravated by subsequent treatments.
\end{abstract}

Keywords Body composition · Cancer pain · Colorectal cancer $\cdot$ Muscle strength $\cdot$ Pain measurement

\section{Introduction}

Paula Postigo-Martin

paulapostigo@ugr.es

1 'Cuídate' From Biomedical Group (BIO277), Instituto de Investigación Biosanitaria Ibs, University of Granada, Granada, Spain

2 Department of Physiotherapy, Faculty of Health Sciences, University of Granada, Granada, Spain

3 Instituto de Investigación Biosanitaria Ibs, GRANADA, Granada, Spain

4 Unit of Excellence On Exercise and Health (UCEES), University of Granada, Granada, Spain
Cancer pain (CP) is one of the most prevalent and concerning aspects of the disease that patients with cancer must face, and it occurs in more than $60 \%$ of patients across all cancer stages [1], even from diagnosis [2]. This pain is very difficult to manage because it is a poorly understood and undertreated syndrome [3] that involves crucial health expenditures [4].

A systematic classification of chronic pain was developed by the International Association for the Study of Pain (IASP) that distinguishes chronic primary and chronic secondary pain syndromes. When pain persists or recurs for more than 3 months, it is considered chronic pain. In some conditions where pain may be considered a disease, the term chronic primary pain is used. However, in other cases, pain is secondary to an underlying disease, such 
as chronic cancer-related pain [5]. Additionally, the term central sensitization is defined by IASP as "increased responsiveness of nociceptive neurons in the central nervous system to their normal or subthreshold afferent input." Clinically, sensitization may only be inferred indirectly from phenomena such as hyperalgesia or allodynia [6].

The presentation of chronic pain and central sensitization in patients with colorectal cancer (CRC) in the survival phase is well established [7,8]. This abnormal processing of nociceptive inputs decreases the pressure pain threshold (PPT) [9]; therefore, low PPT in local and distant areas of cancer reflects primary hyperalgesia and central sensitization, which can increase perceived pain [10]. Depending on its pathogenesis, CP physiopathology may be of nociceptive, neuropathic, mixed, or psychogenic origin. After treatment, a state of central sensitization is increased in $75 \%$ of patients with CRC compared with that in healthy matched controls [8]. Among the possible factors influencing this state are cancer treatments, such as surgery [11], chemotherapy, and radiotherapy [8, 12, 13]; a state of prolonged nociceptive or neuropathic pain [14]; other factors related to muscle and adipose tissue that are closely related to CP $[8,15]$; and certain behaviors in patients, such as kinesiophobia [16], which may increase pain perception.

In patients newly diagnosed with CRC who did not undergo cancer treatment, abdominal pain may already be present [2]. Tumors themselves induce $\mathrm{CP}$ by constricting or invading surrounding tissue, inducing infection or inflammation, or releasing chemicals. Tumor-induced visceral (nociceptive, neuropathic, or mixed) pain can also promote a central sensitization state [14]. However, the psychological distress of the impact of cancer diagnosis (which involves fear, anxiety, pain catastrophizing, and other responses) influences central sensitization and may modulate pain [17] by increasing the level of systemic inflammation through activation of the hypothalamic-pituitary-adrenal axis and sympathetic nervous system [18]. Additionally, these patients present factors related to unhealthy lifestyle habits that are risk factors for CRC appearance [19], which could also be factors that influence the early presentation of $\mathrm{CP}$, as indicated in other populations [20,21].

Although cancer treatment may induce pain, how this may be already established from the moment of diagnosis is unclear. Therefore, it would be interesting to fully elucidate this early $\mathrm{CP}$ appearance to offer tailored interventions to prevent or mitigate $\mathrm{CP}$. Therefore, the current study sought to explore whether $\mathrm{CP}$ already exists in patients with CRC upon diagnosis before cancer treatment compared with patients after treatment and a healthy matched control group. The study also sought to examine whether factors related to the physical health status could influence pain processes.

\section{Methods}

\section{Study design and participants}

We conducted an observational cross-sectional study following the Strengthening the Reporting of Observational Studies in Epidemiology (STROBE) checklist [22]. For this study, the baseline evaluation of two cohorts (newly diagnosed $n=29$; post-treatment $n=40$ ) and 40 healthy age/sex-matched controls were included for comparison. Healthy age/gender-matched controls were recruited through announcements by the University of Granada on social networks. Both previous cohorts had the following inclusion criteria: (1) patients of legal age (>18 years), (2) patients diagnosed with CRC (stage I to IIIa), (3) patients on a waiting list for surgery (newly diagnosed study), or (4) patients completed their medical treatment (post-treatment group). Patients with any medical contraindication or musculoskeletal condition to perform the assessments (e.g., chronic lumbar pain, fibromyalgia, or osteoarthritis), any abdominal surgery, or any previous cancer treatment (newly diagnosed group) were excluded. After the first contact, the patients were contacted by telephone for an appointment at the Sport and Health Research Center or Physiotherapy Laboratory of the Health Science Faculty of the University of Granada. All the participants signed an informed consent form before participating in the study.

The study protocols were approved by the Research Ethics Committee of the University of Granada (0572-M1-16 and 1087-N-16), and the study was performed in accordance with Law 14/2007 on Biomedical Research and the guidelines of the World Medical Association Declaration of Helsinki.

\section{Outcomes}

The same evaluation protocols and assessment instruments (model and brand) were used in all the participants. Evaluations were made by a trained researcher with experience in the evaluation of patients with a $\mathrm{CRC}>3$ years. The patients were asked if they had taken any rescue analgesics in the last $24 \mathrm{~h}$; if so, the assessment could be postponed. The demographic and clinical details were entered from the medical reports of the patients.

\section{Pain}

\section{Pressure pain thresholds (PPT) (kilopascals, kPA)}

Testing was performed using an electronic algometer (Somedic AB. Farsta., Sweden) at the dominant and 
nondominant lumbar, supraumbilical, infraumbilical, and second metacarpal points, with a perpendicular diameter of $1 \mathrm{~cm}$ (absolute value). At each point, the evaluation was performed three times with a rest of $30 \mathrm{~s}$, and progressive increases in force $(30 \mathrm{kPA} / \mathrm{s})$ were applied until the first perception of change from pressure to pain, which was previously explained to the participants. The mean of three rounds was registered as a unidimensional variable with an intraclass correlation coefficient (ICC) of 0.91[23]. Similarly, the "PPT index" (relative PPT value) was calculated in patients with CRC and shows the degree of sensitivity (\%) [12]. This index is obtained by dividing the mean of each PPT point from patients by the mean of each PPT point in the healthy matched control group (HMCG). CRC patients with a higher PPT index were most consistent with HMCG. A difference of $20 \%$ between groups was considered clinically significant [24].

\section{Self-report of spontaneous pain}

Patients were asked to rate their pain intensity in the abdominal and lumbar areas using a horizontal visual analog scale (VAS) of $10 \mathrm{~cm}(\mathrm{~cm})$, where 0 means "no pain" and 10 means "the worst pain." This instrument has an ICC of 0.97 [25]. The cutoff scores for musculoskeletal pain were as follows: mild pain (0 to 3 points), moderate pain ( 3 to 6 points), and severe pain ( $>6$ points) [26].

\section{Abdominal isometric strength}

Abdominal isometric strength was assessed using the trunk curl test to evaluate a possible alteration of the lumbopelvic functional stability. From a supine position with flexion of the knees and hips, patients flexed their trunk to separate the lower angle of the scapula from the stretcher and then maintained this position, with their arms extended without touching their knees as long as they could. Time was recorded up to a maximum of $90 \mathrm{~s}$. This test has a high reliability (ICC $>0.97$ ) [26].

\section{Muscle structure}

Muscle images were captured using an ultrasound device (MyLab 25; Esaote Medical System, Genova, Italy) for the multifidus, transversus abdominis, and external and internal obliques $(\mathrm{cm})$. A 12-MHz linear probe was used following a previous protocol [8]. The images were recollected at a depth of $5 \mathrm{~cm}$ with the patient lying on the stretcher during apnea. The reliability of the ultrasound images for multifidus (ICC $=0.55-0.86)$ and abdominal $($ ICC $>0.81)$ muscle thickness has been previously shown [27].

\section{Body composition and anthropometry}

Body composition, musculoskeletal mass $(\mathrm{kg})$, body fat $(\%)$, body mass index (BMI, $\mathrm{kg} / \mathrm{m}^{2}$ ), and skeletal muscle mass index (musculoskeletal mass/height $2\left(\mathrm{~kg} / \mathrm{m}^{2}\right)$ ) were obtained using an InBody 720 tetrapolar eight-point tactile electrode system (Biospace Co., Ltd., Seoul, Korea). The patients were instructed to rest (no rigorous exercises in the previous $24 \mathrm{~h}$ ) without a meal/water $3 \mathrm{~h}$ before measurement. The cutoff points related to a higher risk of CRC are a weight of $82 \mathrm{~kg}$ and a BMI of $31 \mathrm{~kg} / \mathrm{m}^{2}$ [33]. The skeletal muscle mass index is based on physical disability risk and has been used as a usual cutoff to define moderate sarcopenia when it is between 8.51 and $10.75 \mathrm{~kg} / \mathrm{m}^{2}$ (men) or 5.76 and $6.75 \mathrm{~kg} / \mathrm{m}^{2}$ (women) [28].

Waist circumference $(\mathrm{cm})$ was assessed using plastic tape at the end of exhalation at the midpoint between the lowest rib and iliac crest. A value of $87 \mathrm{~cm}$ is associated with a higher risk of CRC [29].

\section{Statistical analysis and data presentation}

Analyses were performed using the SPSS statistical package for MacOS Sierra version 10.13 (IBM Corp. iReleased 2016, 24.0 version, Armonk, NY: IBM Corp.), with a level of significance of $p<0.05$ and a $95 \%$ confidence interval (CI). The results are expressed as means $(\mathrm{m}) \pm$ standard deviation $(\mathrm{SD})$ for continuous variables or numbers $(n)$ and percentages (\%) for category variables. The Shapiro-Wilk test was used to check the normal distribution of the outcomes $(p>0.05)$. Analysis of variance (ANOVA) was performed to assess the similarity between groups for continuous variables related to demographic and clinical characteristics. The chi-squared $\left(\chi^{2}\right)$ test was used for category variables. Three-way analysis of covariance (ANCOVA) was used to evaluate the betweengroup difference in outcomes with a normal distribution, with ages, stages, and cancer treatment as covariables. Post hoc analysis was performed with the Bonferroni test, and Cohen's $d$ effect size was calculated to quantify the betweengroup differences considered small $(0.20)$, moderate $(0.50)$, and large (0.80). The Kruskal-Wallis test was used when the outcomes did not reach normality, and post hoc comparisons were performed using the Dunn-Bonferroni post hoc method. Hedge's effect size was calculated to quantify the between-group differences, which were considered small (0.20), moderate (0.50), and large (0.80). Additionally, Pearson's test was used to analyze the bivariate correlation between the dominant lumbar side of the PPT and the remaining dependent outcomes in each group. A correlation from 0 to 0.25 indicates an absent or weak relationship, a correlation from 0.25 to 0.50 indicates a fair relationship, a correlation from 0.50 to 0.75 indicates a moderate to good relationship, and a correlation greater than 0.75 indicates a 
very good relationship [30]. Missing data were not included in the analysis.

\section{Results}

Of the 239 screened patients, 110 were eligible to complete the assessment. The reasons for ineligibility included participation declination $(n=76)$, not meeting the inclusion or exclusion criteria $(n=46)$, and failure in the assessment instruments $(n=7)$. Finally, 29 patients $(69.0 \%$ men) with an average age of $61.68 \pm 12.78$ years were included in the newly diagnosed group (NDG), 40 patients $(65.0 \%$ men) with an average age of $60.80 \pm 10.02$ years were included in the post-treatment group (PTG), and 40 healthy matched people $(52.5 \%$ men) with an average age of $59.54 \pm 9.69$ years were included in the HMCG. The demographic and clinical characteristics of each participant group are shown in Table 1.

\section{Pain}

Figure 1 shows the PPT differences between groups. ANCOVA detected significant differences between groups at all PPT evaluation points: lumbar side (dominant; $F=5.4$, $p=0.006$; nondominant; $F=12.2, p<0.001$ ), supraumbilical side (dominant; $F=10.8, p<0.001$; nondominant; $F=10.8, p<0.001$ ), infraumbilical side (dominant; $F=7.8$, $p=0.001$; nondominant; $F=8.0 p=0.001)$, and second metacarpal side (dominant; $F=5.5, p=0.005$; nondominant; $F=7.7, p=0.001)$. The NDG and PTG registered lower values than the HMCG and were always lower in the PTG. The intergroup effect size between the NDG and PTG was large for the supraumbilical dominant side $(d=0.81$; $\mathrm{CI}=0.29,1.32)$ and moderate for the lumbar nondominant side $(d=0.57 ; \mathrm{CI}=0.07,1.06)$, supraumbilical nondominant side $(d=0.57 ; \mathrm{CI}=0.07,1.07)$, infraumbilical dominant side $(d=0.61 ; \mathrm{CI}=0.11,1.10)$, and infraumbilical nondominant side $(d=0.51 ; \mathrm{CI}=0.01,0.99)$. The intergroup effect size between the NDG and HMCG was moderate for

Table 1 Demographic and clinical characteristics of the groups

\begin{tabular}{|c|c|c|c|c|c|}
\hline \multirow{2}{*}{$\overline{\text { Age (years) } \mathrm{m} \pm \mathrm{SD}}$} & & \multirow{2}{*}{$\begin{array}{l}\text { Newly diagnosed }(n=29) \\
61.68 \pm 12.781\end{array}$} & \multirow{2}{*}{$\begin{array}{l}\text { Post-treatment }(n=40) \\
60.80 \pm 10.02\end{array}$} & \multirow{2}{*}{$\begin{array}{l}\text { Healthy matched } \\
\text { control }(n=40)\end{array}$} & \multirow{2}{*}{$\frac{p \text { value }}{0.712}$} \\
\hline & & & & & \\
\hline \multicolumn{2}{|c|}{ Time since surgery (months) $\mathrm{m} \pm \mathrm{SD}$} & - & $13.26 \pm 8.76$ & - & - \\
\hline \multirow{2}{*}{$\begin{array}{l}\text { Gender } \\
n(\%)\end{array}$} & Male & $20(69.0)$ & $26(65.0)$ & $21(52.5)$ & \multirow[t]{2}{*}{0.323} \\
\hline & Female & $9(31.0)$ & $14(35.0)$ & $19(47.5)$ & \\
\hline \multirow{4}{*}{$\begin{array}{l}\text { Social situation } \\
n(\%)\end{array}$} & Single & $2(6.9)$ & $3(7.5)$ & $2(5.0)$ & \multirow[t]{4}{*}{0.101} \\
\hline & Married & $22(75.9)$ & $34(85.0)$ & $30(75.0)$ & \\
\hline & Divorced & $1(3.4)$ & $1(2.5)$ & $7(17.5)$ & \\
\hline & Widowed & $4(13.8)$ & $2(5.0)$ & $1(2.5)$ & \\
\hline \multirow{3}{*}{$\begin{array}{l}\text { Smoking status } \\
n(\%)\end{array}$} & Never smoked & $13(44.8)$ & $20(50.0)$ & $20(50.0)$ & \multirow[t]{3}{*}{0.988} \\
\hline & Current smoker & $3(10.3)$ & $4(10.0)$ & $4(10.0)$ & \\
\hline & Ex smoker & $13(44.8)$ & $16(40.0)$ & $15(37.5)$ & \\
\hline \multirow{4}{*}{$\begin{array}{l}\text { Alcohol intake } \\
n(\%)\end{array}$} & Never & $15(51.7)$ & $15(37.5)$ & $14(35.0)$ & \multirow[t]{4}{*}{0.330} \\
\hline & Monthly & $4(13.8)$ & $9(22.5)$ & $6(15.0)$ & \\
\hline & Weekly & $2(6.9)$ & $9(22.5)$ & $11(27.5)$ & \\
\hline & Daily & $8(27.6)$ & $7(17.5)$ & $7(17.5)$ & \\
\hline $\begin{array}{l}\text { Physical activity level } \\
n(\%)\end{array}$ & $\begin{array}{l}<10 \mathrm{MET} / \mathrm{h} \mathrm{w} \\
>10 \mathrm{MET} / \mathrm{h} \text { week }\end{array}$ & $\begin{array}{l}2(7.7) \\
24(92.3)\end{array}$ & $\begin{array}{l}5(12.5) \\
35(87.5)\end{array}$ & $\begin{array}{l}3(8.3) \\
33(91.7)\end{array}$ & - \\
\hline \multirow{3}{*}{$\begin{array}{l}\text { Cancer stage } \\
n(\%)\end{array}$} & I & $5(17.2)$ & $0(0.0)$ & - & \multirow[t]{3}{*}{-} \\
\hline & II & $5(17.2)$ & $14(35.0)$ & - & \\
\hline & III & $16(55.2)$ & $25(62.5)$ & - & \\
\hline \multirow[t]{4}{*}{ Medical treatment } & No treatment & $29(100)$ & $8(20.0)$ & $40(100)$ & \multirow[t]{4}{*}{-} \\
\hline & Radiotherapy & - & $3(7.5)$ & - & \\
\hline & Chemotherapy & - & $16(40.0)$ & - & \\
\hline & $\begin{array}{l}\text { Radiotherapy and } \\
\text { chemotherapy }\end{array}$ & - & $13(32.5)$ & - & \\
\hline
\end{tabular}

$p$ values of between-group differences using ANOVA test for independent samples (continuous variables) and $X^{2}$ analysis (categorical variables). $m$, mean; $n$, sample size; $S D$, standard deviation; \% (percentage). ${ }^{*} p<0.05 ; * * p<0.001$ 


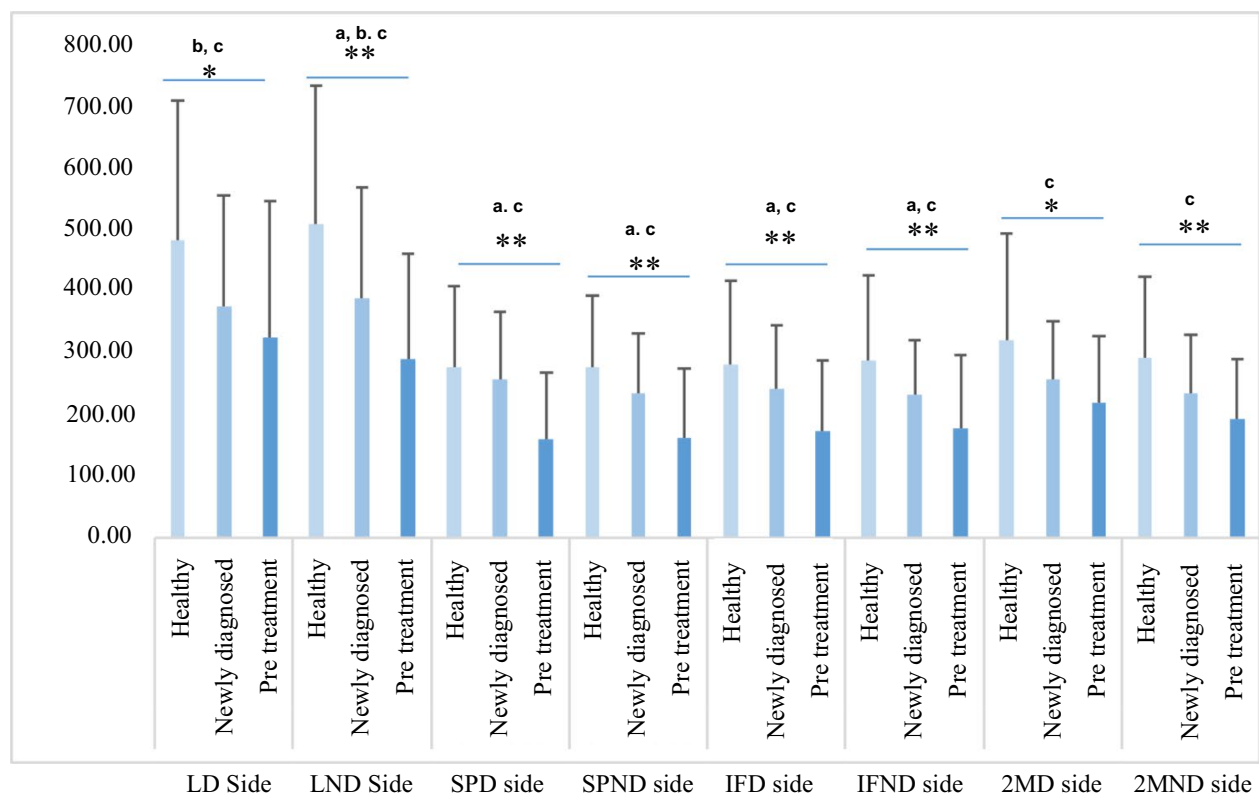

Fig. 1 Pressure pain thresholds $(\mathrm{kPa})$ between-groups differences. ${ }^{*} p<0.05 ;{ }^{*} p<0.001 ;$ a - between newly diagnosed and posttreatment groups differences with Bonferroni post hoc; b-between newly diagnosed and healthy matched control groups differences with Bonferroni post hoc; c-between post-treatment and healthy matched control groups differences with Bonferroni post hoc. LD side, lumbar dominant side; LND side, lumbar nondominant side; SPD side, supraumbilical dominant side; SPND side, supraumbilical nondominant side; IFD side, infraumbilical dominant side; IFND side, infraumbilical nondominant side; $2 \mathrm{MD}$ side, second metacarpal dominant side; $2 \mathrm{MND}$ side, second metacarpal nondominant side

dominant side $(n=15,53.5 \%$ in the NDG; $n=27,67.5 \%$ in the PTG), and second metacarpal nondominant side ( $n=17,66.7 \%$ in the NDG; $n=27,67.5 \%$ in the PTG). Figure 2 shows PPT index differences between NDG and PTG.

The Kruskal-Wallis test of self-reported spontaneous pain revealed a significant difference between groups in abdominal pain $(p=0.006)$. Figure 3 shows differences in VAS $(\mathrm{cm})$ at the abdominal and lumbar areas between groups. The post hoc analysis identified significant differences between the NDG and HMCG $(p=0.005)$. The intergroup effect size was moderate $(g=0.90 ; \mathrm{CI}=0.39,1.40)$ between these groups (Fig. 3). No significant differences were found in lumbar pain $(p=0.920)$.

\section{Abdominal isometric strength}

The Kruskal-Wallis test revealed a significant difference $(p<0.001)$ between groups for abdominal isometric strength, with lower values in the NDG and PTG than in the HMCG. Post hoc analysis identified significant differences between the NDG and HMCG $(p=0.011)$ and between the PTG and HMCG $(p<0.001)$. Table 2 shows comparisons between groups according to the abdominal isometric strength. 


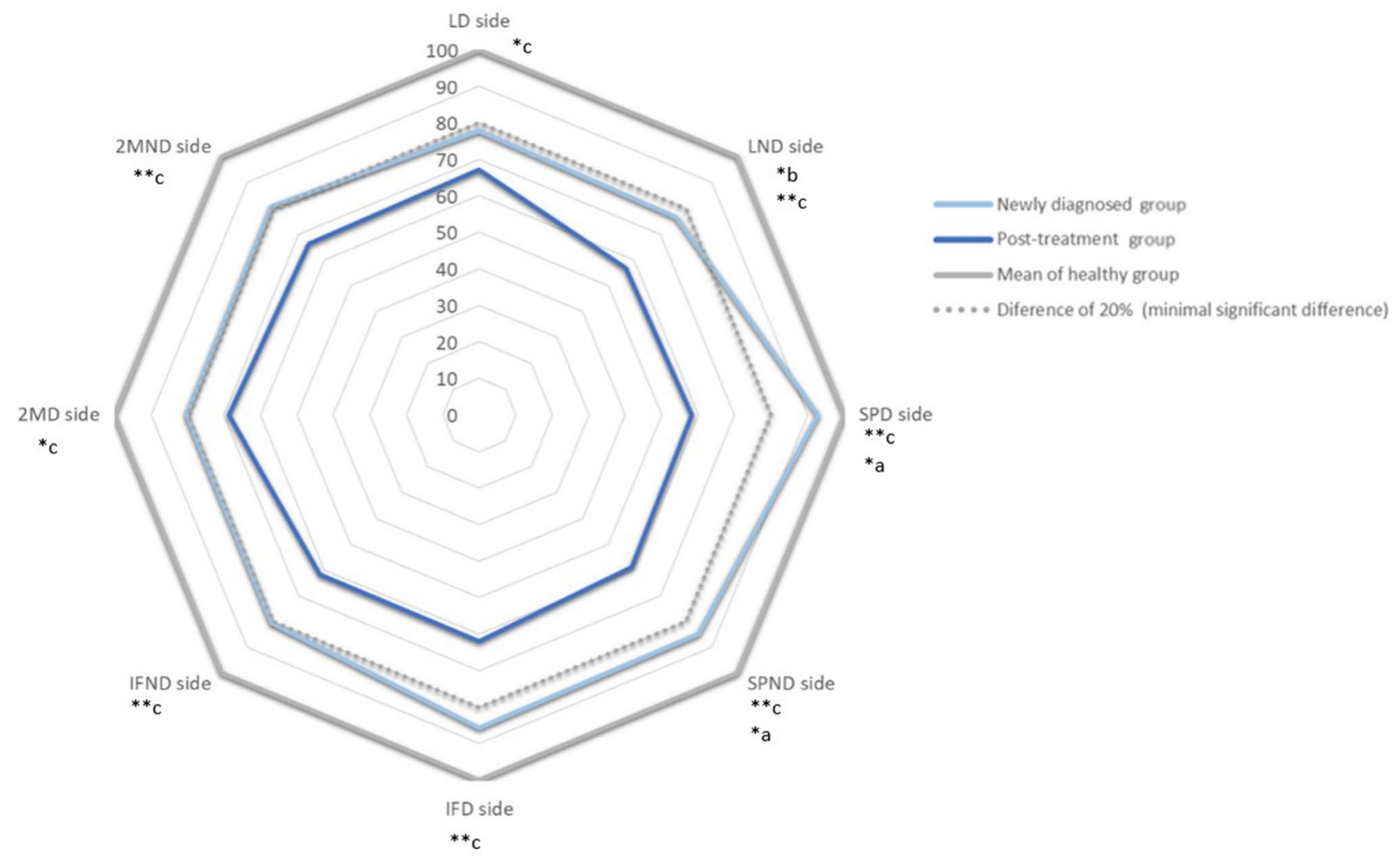

Fig. 2 Pressure pain threshold index differences between newly diagnosed and post-treatment groups. $* p<0.05$; ** $p<0.001$; a-between newly diagnosed and healthy matched control group differences with Bonferroni post hoc; $\mathrm{b}$ - between healthy matched control and posttreatment groups differences with Bonferroni post hoc; c-between newly diagnosed and post-treatment groups differences with Bonfer- roni post hoc; LD side, lumbar dominant side; LND side, lumbar nondominant side; SPD side, supraumbilical dominant side; SPND side, supraumbilical nondominant side; IFD side, infraumbilical dominant side; IFND side, infraumbilical nondominant side; $2 \mathrm{MD}$ side, second metacarpal dominant side; $2 \mathrm{MND}$ side, second metacarpal nondominant side
Fig. 3 Between groups differences in VAS $(\mathrm{cm})$ at abdominal and lumbar area. HMCG, healthy matched control group; NDG, newly diagnosed group; PTG, post-treatment group. ${ }^{*} p<0.05$ with the KruskalWallis test

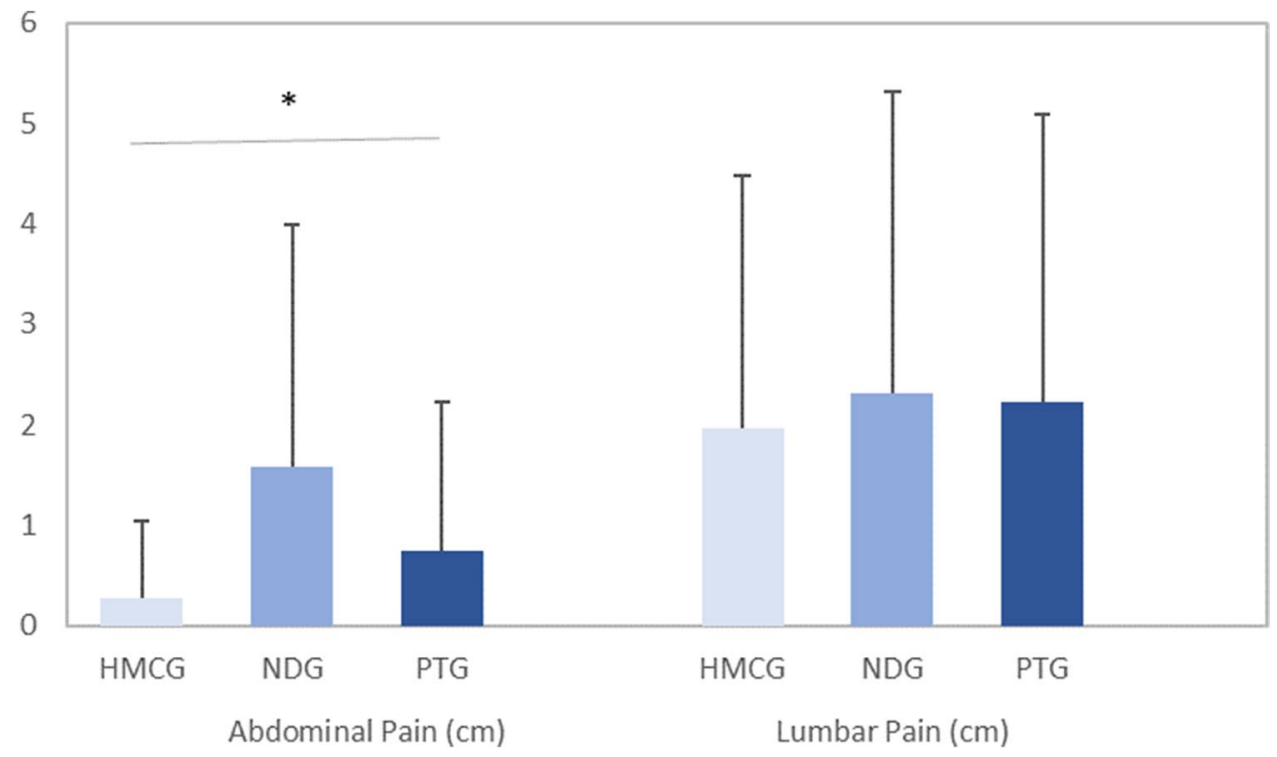




\section{Muscle structure}

The Kruskal-Wallis test showed a significant difference in the width of the lumbar multifidus $(p<0.002)$ between groups, with lower values in the NDG and PTG than in the HMCG. Post hoc analysis identified significant differences between the NDG and HMCG $(p=0.011)$ and between the PTG and HMCG $(p=0.004)$. Table 2 shows the comparison between groups according to muscle structure.

\section{Body composition and anthropometric outcomes}

ANCOVA of musculoskeletal mass data revealed a significant difference between groups $(F=3.14 ; p=0.047)$, with lower values in the PTG than in the NDG and HMCG. Bonferroni post hoc analysis identified significant differences between the NDG and PTG $(p=0.014$; $\mathrm{CI}=0.76,6.65$ ). Additionally, the Kruskal-Wallis test showed a significant difference between groups for the skeletal muscle mass index $(p=0.038)$. Post hoc analysis identified significant differences between the NDG and PTG $(p=0.042)$. No significant differences were found for the remaining variables. Table 2 shows comparisons between groups according to body composition and anthropometric outcomes.

\section{Correlations}

In all groups, Pearson's test showed a significant positive association $(p<0.001)$ between the dominant lumbar side point and remaining PPT points. In the PTG and HMCG, positive associations were observed between the dominant lumbar side points and abdominal isometric strength $(r s=0.471$ and $p=0.002$ in the PTG; $r s=0.501$ and $p=0.003$ in the HMCG) and musculoskeletal mass ( $r s=0.320$ and $p=0.044$ in the PTG; $r s=0.548$ and $p=0.001$ in the HMCG). Additionally, negative associations were observed between the dominant lumbar side points and body fat $(r s=-0.390$ and $p=0.013$ in the PTG; $r s=-0.429$ and $p=0.010$ in the HMCG). Figure 4 shows a schematic representation of the bivariate correlation between the lumbar dominant side of the PPT and remaining dependent outcomes in each group.

\section{Discussion}

We found that CP is already present in CRC patients at diagnosis prior to treatment. The analysis detected a threshold reduction in most PPT points, lower values in the PPT index, higher self-reported abdominal pain, and lower abdominal strength in newly diagnosed patients, with even more marked results in post-treatment patients, where lower lean mass and skeletal muscle index values were also found compared with those in the healthy matched controls.

Curiously, our findings of a reduction in PPT suggest that from the moment of diagnosis, patients with CRC had CP, indicating the possible onset of central sensitization without the presence of some of the factors that may enhance $\mathrm{CP}$ in the post-treatment group. Additionally, 1 of 2 patients in the NDG showed a minimal clinical difference $(>20 \%)$ in the PPT index compared with that in the HMCG. There are studies that address the issue of pain in newly diagnosed cancer patients, although their cohort is only partially treatment-naïve and it does not focus on patients with CRC. In the study by Ger et al. [31], a Taiwanese cohort of patients newly diagnosed with several types of cancer, including CRC, was analyzed. They found that $38 \%(n=113)$ of the patients presented $\mathrm{CP}$, and that only in $8 \%$ of those cases was due to cancer treatment. Also, they found that, among other reasons, pain prevalence correlated with patient socioeconomic characteristics (i.e., lower medical insurance coverage) and pain severity with a more advanced stage of the disease and previous inadequate pain management. In another study by Kelsen et al. [32], they analyzed data from newly diagnosed (64\%), and just after their first chemotherapy (36\%) patients with pancreas cancer. They found that there was a percentage having none (37\%), mild (34\%), or moderate-severe (29\%) pain. Also, that their cohort presented less pain among the preoperative patients, but also that there was a correlation between depressive symptoms and pain (which $38 \%$ of the cohort presented). These results could show the influence from both physiological and psychosocial dimensions of pain [33], which are sometimes present at diagnosis.

Additionally, the isometric strength values were 30\% lower in the NDG and almost $60 \%$ lower in the PTG than in the HMCG, findings that are consistent with other study findings from our research group on PTG patients [34, 35]. The lower abdominal strength in NDG patients was a negative finding and shows the possible loss of muscle strength that often accompanies chronic pain [36]. Furthermore, the lumbopelvic area is the central area of the body where muscle chains are located [37, 38]. Functional alteration of the area could be related to a greater possibility of sacral fractures [39], joint instabilities [40], and low back pain [41, 42]. Additionally, previous evidence has shown that NDG earlystage patients with CRC already show muscle dysfunction, a phenomenon considered undetected in clinical practice but that shows a strong association with vital clinical end points, including survival and treatment toxicity [43]. Such findings could be used to start programs focused on strength exercises from diagnosis to try to mitigate the detrimental effects of future treatments on muscle strength.

Related to general muscle mass, the skeletal muscle mass index indicated that only the PTG showed moderate 


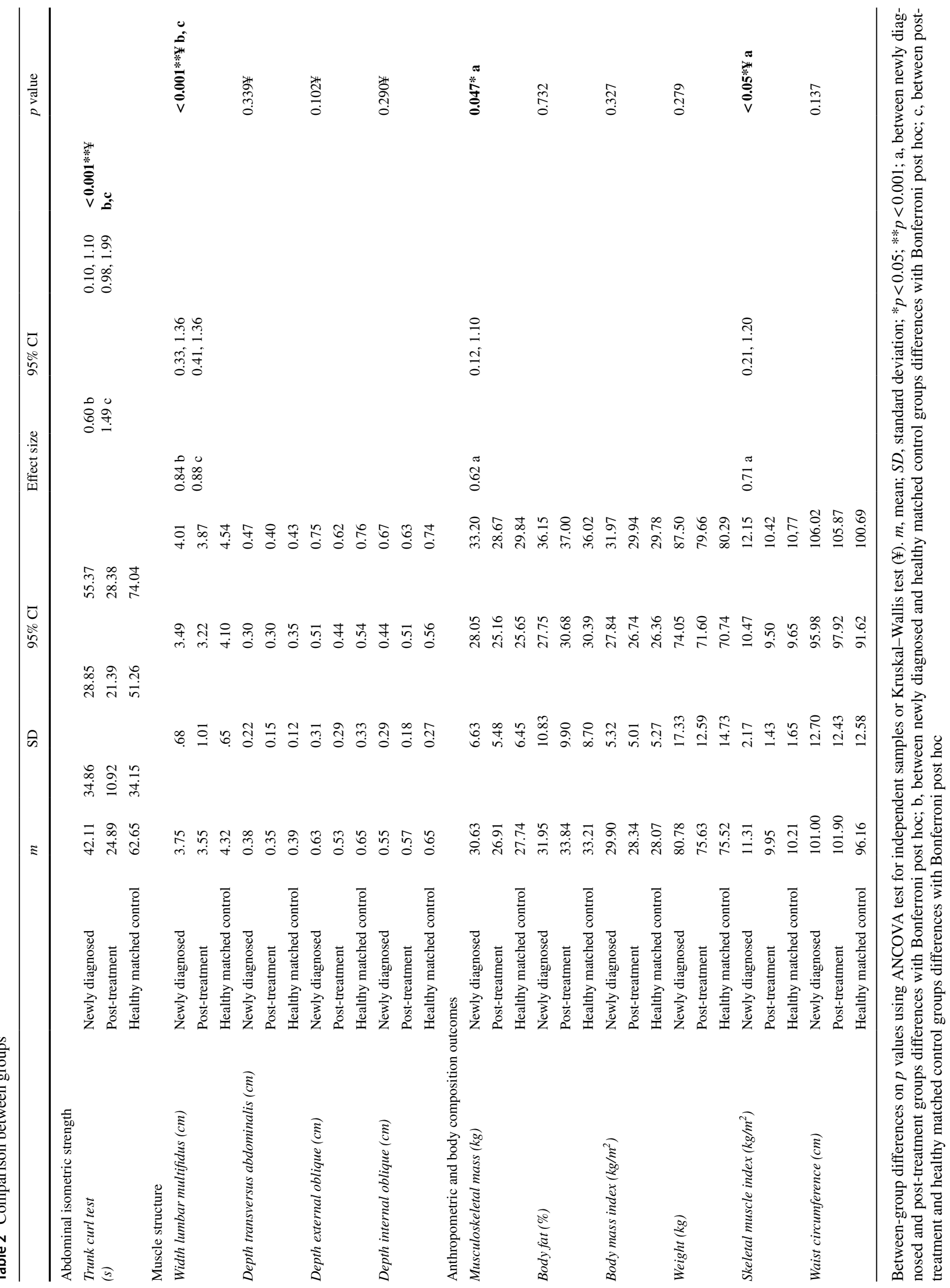




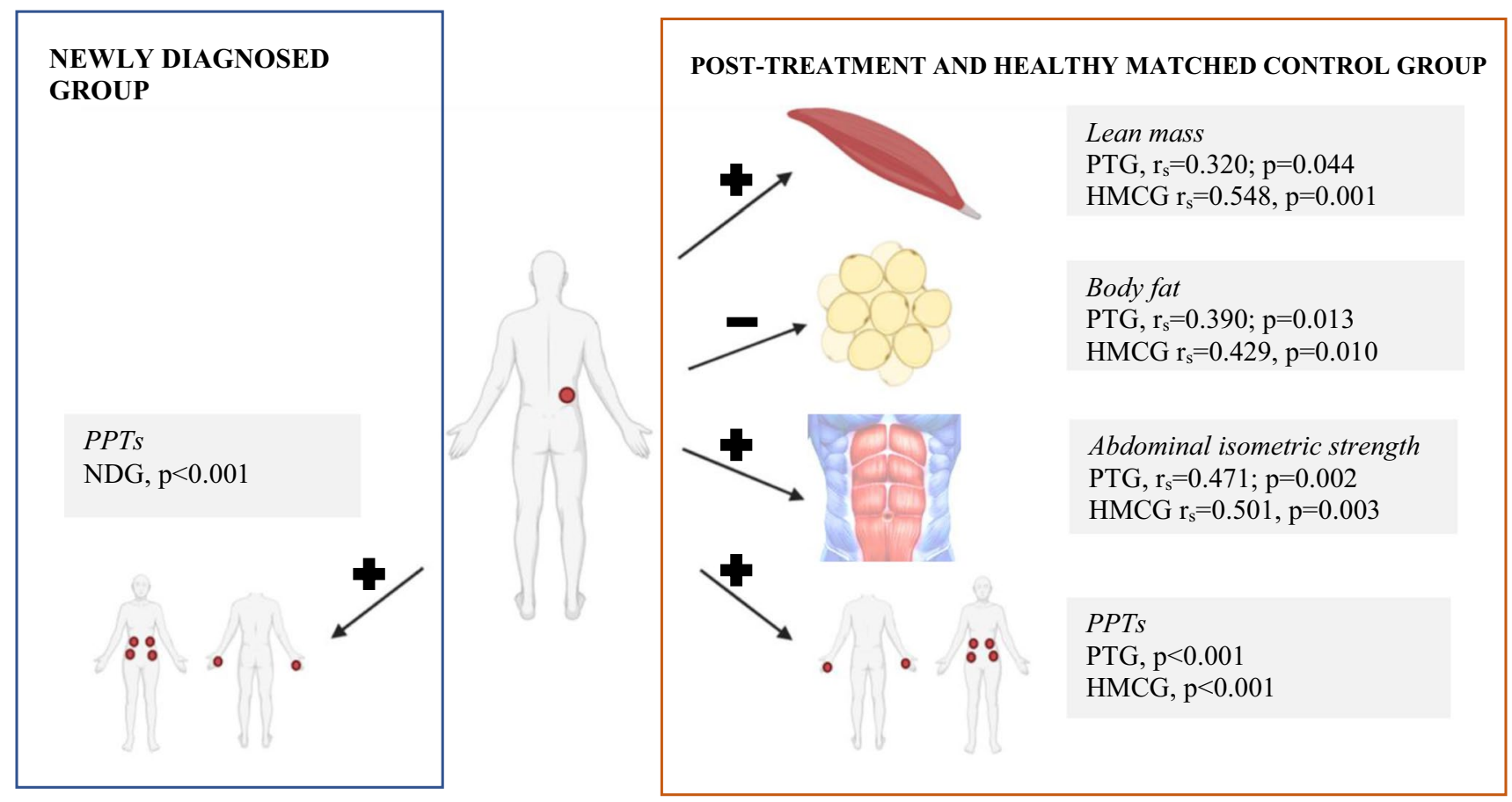

Fig. 4 Bivariate correlation between lumbar dominant side of pressure pain threshold and the rest of dependent outcomes in each group. Created in BioRender.com. + positive correlation;-negative corre-

sarcopenia, a prevalent problem in patients with cancer because it involves a higher risk of developing immediate postoperative complications and decreased tolerance to chemoradiotherapy because of side effects [44]. However, in the muscles around the tumor, both the NDG and PTG presented a width reduction (with $13.19 \%$ less lumbar multifidus width in the NDG and $17.82 \%$ in the PTG) compared with the HMCG, a finding that is consistent with previous findings in patients with CRC $[15,45]$. This early impact in muscle close to the tumor location could be caused by tumor inflammation-released cytokines [46]. Additionally, multifidus reduction may be related to overall survival [47], and its dysfunction is strongly associated with chronic low back pain [48].

Correlation analysis revealed that the PTG and HMCG were unexpectedly similar, with reductions in the dominant lumbar side PPT correlated with the remaining PPT points, lower values of isometric abdominal strength, lean mass, and higher body fat in both groups. Better muscle function may mitigate pain perception [49, 50]; additionally, a lower PPT is related to an excessive fat percentage, which is also associated with body biomechanical/structural changes, increased inflammatory mediators, mood disturbance, poor sleep, and lifestyle issues [21], which may explain our findings. In the NDG, these correlations did not appear except for among the PPT points, and our algometry data in the NDG showed data dispersion. Therefore, we supposed that the wide range lation. LDS, lumbar dominant side; NDG, newly diagnosed group; PPTs, pressure pain thresholds; PTG, post-treatment group; HMCG, healthy matched control group

of variable responses might be due to the impact of the diagnosis. These findings highlight the importance of considering body composition, specifically increasing muscle and decreasing adipose tissue, in the pain management of these patients because it may indirectly affect their pain. In the case of newly diagnosed patients, body composition could help prevent this situation; however, additional studies are needed to clarify these findings.

Some limitations of our study should be noted. First, not all the factors that influence the development of central sensitization from the biopsychosocial perspective were analyzed in these patients; secondly, analyses with different groups limit the results, and no longitudinal changes could be studied; also, the study did not examine the presence of background pain or record any analgesic treatment; therefore, these characteristics were not established as inclusion criteria to establish a representative sample of patients with CRC.

This study also presents some strengths. Widespread pain, which is a crucial objective measure, was addressed. Also, this work attempts to respond to the limitations of a previous study in which prospective data from patients with $\mathrm{CRC}$ was needed to be obtained upon diagnosis [8]. Moreover, it highlights the deterioration of the health status at the time of diagnosis, thus reinforcing the need for multidisciplinary interventions that are necessary and must include, in addition to multimodal physical exercise interventions 
(endurance, resistance, strength, motor control, and flexibility, among others), educational, nutritional, and psychosocial support interventions [51].

\section{Conclusion}

Before the start of cancer treatment, NDG patients with CRC show signs of primary hyperalgesia, central sensitization, and deterioration in physical condition and body composition. Such symptoms appear to be further aggravated following cancer treatment. Hence, addressing the health status of these patients at diagnosis is crucial.

Author contribution ICV, MAM, and AAO conceptualized the study and wrote the manuscript. PPM, MLG, and AGS performed statistical analyses and wrote the manuscript. AMFP recruited and measured patients with cancer and healthy matched controls. AMFP and AAO created the database. All the authors analyzed and interpreted the data and revised and edited the manuscript for submission.

Funding Funding for open access charge: Universidad de Granada / CBUA. This study is funded by the Spanish Ministry of Education Culture and Sport (MECD) (FPU17/00939, FPU18/03575), the European Social Fund, the Carlos III Health Institute (FI19/00230), and the CEIBiotic Universidad de Granada (CEI13-MP18 and CEI14-MPBS40).

Availability of data and material Data will be available upon request from the corresponding author.

\section{Declarations}

Ethics approval The study protocols were approved by the Research Ethics Committee of the University of Granada (0572-M1-16 and 1087-N-16), and the study was performed in accordance with Law 14/2007 on Biomedical Research and the guidelines of the WMA Declaration of Helsinki.

Consent to participate All the participants were informed verbally and in writing before signing the informed consent form.

Consent for publication Not applicable.

Conflict of interest The authors declare no competing interests.

Open Access This article is licensed under a Creative Commons Attribution 4.0 International License, which permits use, sharing, adaptation, distribution and reproduction in any medium or format, as long as you give appropriate credit to the original author(s) and the source, provide a link to the Creative Commons licence, and indicate if changes were made. The images or other third party material in this article are included in the article's Creative Commons licence, unless indicated otherwise in a credit line to the material. If material is not included in the article's Creative Commons licence and your intended use is not permitted by statutory regulation or exceeds the permitted use, you will need to obtain permission directly from the copyright holder. To view a copy of this licence, visit http://creativecommons.org/licenses/by/4.0/.

\section{References}

1. van den Beuken-van Everdingen MHJ, de Rijke JM, Kessels AG et al (2007) Prevalence of pain in patients with cancer: a systematic review of the past 40 years. Ann Oncol Off J Eur Soc Med Oncol 18:1437-1449. https://doi.org/10.1093/annonc/mdm056

2. Astin M, Griffin T, Neal RD et al (2011) The diagnostic value of symptoms for colorectal cancer in primary care: a systematic review. Br J Gen Pract 61(586):e231-43. https://doi.org/10.3399/ bjgp11X572427

3. Gress KL, Charipova K, Kaye AD et al (2020) An overview of current recommendations and options for the management of cancer pain: a comprehensive review. Oncol Ther 8:251. https://doi. org/10.1007/S40487-020-00128-Y

4. Fortner BV, Demarco G, Irving G et al (2003) Description and predictors of direct and indirect costs of pain reported by cancer patients. J Pain Symptom Manage 25:9-18. https://doi.org/10. 1016/s0885-3924(02)00597-3

5. Treede RD, Rief W, Barke A et al (2019) Chronic pain as a symptom or a disease: the IASP classification of chronic pain for the International Classification of Diseases (ICD-11). Pain 160:1927. https://doi.org/10.1097/j.pain.0000000000001384

6. Terminology: International Association for the Study of Pain. International Association for the Study of Pain (IASP). 2021. https://www.iasp-pain.org/resources/terminology/. Accessed 5 Nov 2021

7. Campbell KL, Winters-Stone KM, Wiskemann J et al (2019) Exercise guidelines for cancer survivors: consensus statement from international multidisciplinary roundtable. Med Sci Sports Exerc 51:2375-2390. https://doi.org/10.1249/MSS.0000000000002116

8. Sánchez-Jiménez A, Cantarero-Villanueva I, Molina-Barea R et al (2014) Widespread pressure pain hypersensitivity and ultrasound imaging evaluation of abdominal area after colon cancer treatment. Pain Med (United States) 15:233-240. https://doi.org/10. 1111/pme.12281

9. Özdolap Ş, Sarikaya S, Köktürk F (2014) Evaluation of pain pressure threshold and widespread pain in chronic low back pain. Turkiye Fiz Tip ve Rehabil Derg 60:32-36. https://doi.org/10.5152/ tftrd.2014.71602

10. De Groef A, Meeus M, De Vrieze T et al (2018) Unraveling selfreported signs of central sensitization in breast cancer survivors with upper limb pain: prevalence rate and contributing factors. Pain Physician 21:E247-E256

11. Van de Ven TJ, John Hsia H-L (2012) Causes and prevention of chronic postsurgical pain. Curr Opin Crit Care 18:366-371. https://doi.org/10.1097/MCC.0b013e3283557a7f

12. Fernández-Lao C, Cantarero-Villanueva I, Fernández-De-LasPeñas C et al (2011) Widespread mechanical pain hypersensitivity as a sign of central sensitization after breast cancer surgery: comparison between mastectomy and lumpectomy. Pain Med 12:72-78. https://doi.org/10.1111/j.1526-4637.2010.01027.x

13. Caro-Morán E, Díaz-Rodríguez L, Cantarero-Villanueva I et al (2014) Nerve pressure pain hypersensitivity and upper limb mechanosensitivity in breast cancer survivors: a case-control study. Pain Med 15:1715-1723. https://doi.org/10.1111/pme.12567 
14. Ashmawi HA, Freire GMG (2016) Peripheral and central sensitization Rev Dor 17:31-34. https://doi.org/10.5935/1806-0013. 20160044

15. Cantarero-Villanueva I, Cuesta-Vargas AI, Lozano-Lozano M et al (2020) Changes in pain and muscle architecture in colon cancer survivors after a lumbopelvic exercise program: a secondary analysis of a randomized controlled trial. Pain Med (United States) 18:1366-1376. https://doi.org/10.1093/pm/pnx026

16. Eng L, Pringle D, Su J et al (2018) Patterns, perceptions, and perceived barriers to physical activity in adult cancer survivors. Support Care Cancer 26:3755-3763. https://doi.org/10.1007/ s00520-018-4239-5

17. Magee D, Bachtold S, Brown M, Farquhar-Smith P (2019) Cancer pain: where are we now? Pain Manag 9:63-79. https://doi.org/10. 2217/pmt-2018-0031

18. Powell ND, Tarr AJ, Sheridan JF (2013) Psychosocial stress and inflammation in cancer. Brain Behav Immun 30(Suppl):S41-S47. https://doi.org/10.1016/j.bbi.2012.06.015

19. Nunez C, Nair-Shalliker V, Egger S et al (2018) Physical activity, obesity and sedentary behaviour and the risks of colon and rectal cancers in the 45 and up study. BMC Public Health 18:325. https://doi.org/10.1186/s12889-018-5225-Z

20. Pinto RZ, Ferreira PH, Kongsted A et al (2014) Self-reported moderate-to-vigorous leisure time physical activity predicts less pain and disability over 12 months in chronic and persistent low back pain. Eur J Pain 18:1190-1198. https://doi.org/10.1002/j. 1532-2149.2014.00468.x

21. Okifuji A, Hare BD (2015) The association between chronic pain and obesity. J Pain Res 8:399-408. https://doi.org/10.2147/JPR. S55598

22. Vandenbroucke JP, von Elm E, Altman DG et al (2014) Strengthening the Reporting of Observational Studies in Epidemiology (STROBE): explanation and elaboration. Int J Surg 12:1500 1524. https://doi.org/10.1016/j.ijsu.2014.07.014

23. Chesterton LS, Sim J, Wright CC, Foster NE (2007) Interrater reliability of algometry in measuring pressure pain thresholds in healthy humans, using multiple raters. Clin J Pain 23:760-766. https://doi.org/10.1097/AJP.0b013e318154b6ae

24. Prushansky T, Dvir Z, Defrin-Assa R (2004) Reproducibility indices applied to cervical pressure pain threshold measurements in healthy subjects. Clin J Pain 20:341-347. https://doi.org/10.1097/ 00002508-200409000-00009

25. Bijur PE, Silver W, Gallagher EJ (2001) Reliability of the visual analog scale for measurement of acute pain. Acad Emerg Med 8:1153-1157. https://doi.org/10.1111/j.1553-2712.2001.tb011 32.x

26. Olufade T, Gallicchio L, MacDonald R, Helzlsouer KJ (2015) Musculoskeletal pain and health-related quality of life among breast cancer patients treated with aromatase inhibitors. Support care cancer Off J Multinatl Assoc Support Care Cancer 23:447455. https://doi.org/10.1007/s00520-014-2364-3

27. Cuellar WA, Blizzard L, Callisaya ML et al (2017) Test-retest reliability of measurements of abdominal and multifidus muscles using ultrasound imaging in adults aged 50-79 years. Musculoskelet Sci Pract 28:79-84. https://doi.org/10.1016/j.msksp.2016. 11.013

28. Janssen I, Baumgartner RN, Ross R et al (2004) Skeletal muscle cutpoints associated with elevated physical disability risk in older men and women. Am J Epidemiol 159:413-421. https://doi.org/ 10.1093/aje/kwh058

29. Heo M, Kabat GC, Strickler HD et al (2015) Optimal cutoffs of obesity measures in relation to cancer risk in postmenopausal women in the Women's Health Initiative Study. J Womens Health (Larchmt) 24:218-227. https://doi.org/10.1089/jwh.2014.4977

30. Colton T (1974) Statistics in medicine. Little, Brown and Company, Boston

31. Ger L-P, Ho S-T, Wang J-J, Cherng C-H (1998) The prevalence and severity of cancer pain. J Pain Symptom Manage 15:285-293. https://doi.org/10.1016/S0885-3924(98)00017-7

32. Kelsen DP, Portenoy RK, Thaler HT et al (1995) Pain and depression in patients with newly diagnosed pancreas cancer. J Clin Oncol 13:748-755. https://doi.org/10.1200/JCO.1995.13.3.748

33. Adams LM, Turk DC (2018) Central sensitization and the biopsychosocial approach to understanding pain. J Appl Biobehav Res 23:e12125. https://doi.org/10.1111/jabr.12125

34. Cantarero-Villanueva I, Fernández-Lao C, Cuesta-Vargas AI et al (2013) The effectiveness of a deep water aquatic exercise program in cancer-related fatigue in breast cancer survivors: a randomized controlled trial. Arch Phys Med Rehabil 94:221-230. https://doi. org/10.1016/j.apmr.2012.09.008

35. Cantarero-Villanueva I, Sánchez-Jiménez A, Galiano-Castillo N et al (2016) Effectiveness of lumbopelvic exercise in colon cancer survivors. Med Sci Sport Exerc 48:1438-1446. https://doi.org/10. 1249/MSS.0000000000000917

36. Nourbakhsh MR, Arab AM (2002) Relationship between mechanical factors and incidence of low back pain. 102519/ jospt2002329447 32:447-460. https://doi.org/10.2519/JOSPT. 2002.32.9.447

37. Chu SK, Jayabalan P, Ben KW, Press J (2016) The kinetic chain revisited: new concepts on throwing mechanics and injury. PM R 8:S69-77. https://doi.org/10.1016/j.pmrj.2015. 11.015

38. Putnam CA (1993) Sequential motions of body segments in striking and throwing skills: descriptions and explanations. J Biomech 26(Suppl 1):125-135. https://doi.org/10.1016/0021-9290(93) 90084-r

39. Bishop JA, Dangelmajer S, Corcoran-Schwartz I et al (2017) Bilateral sacral ala fractures are strongly associated with lumbopelvic instability. J Orthop Trauma 31:636-639. https://doi.org/ 10.1097/BOT.0000000000000972

40. McCann RS, Johnson K, Suttmiller AMB (2021) Lumbopelvic stability and trunk muscle contractility of individuals with chronic ankle instability. Int J Sports Phys Ther 16:741-748. https://doi. org/10.26603/001c.22132

41. Hodges PW, Moseley GL (2003) Pain and motor control of the lumbopelvic region: effect and possible mechanisms. J Electromyogr Kinesiol Off J Int Soc Electrophysiol Kinesiol 13:361-370. https://doi.org/10.1016/s1050-6411(03)00042-7

42. Hodges PW, Richardson CA (1996) Inefficient muscular stabilization of the lumbar spine associated with low back pain. A motor control evaluation of transversus abdominis. Spine (Phila Pa 1976) 21:2640-2650. https://doi.org/10.1097/00007632-19961 1150-00014

43. Christensen JF, Jones LW, Andersen JL et al (2014) Muscle dysfunction in cancer patients. Ann Oncol 25:947-958. https://doi. org/10.1093/annonc/mdt551

44. Prado CMM, Lima ISF, Baracos VE et al (2011) An exploratory study of body composition as a determinant of epirubicin pharmacokinetics and toxicity. Cancer Chemother Pharmacol 67:93-101. https://doi.org/10.1007/s00280-010-1288-y

45. Cruz-Fernández M, Achalandabaso-Ochoa A, Gallart-Aragón T et al (2020) Quantity and quality of muscle in patients recently diagnosed with colorectal cancer: a comparison with cancer-free controls. Support Care Cancer 28:4745-4752. https://doi.org/10. 1007/s00520-020-05314-2 
46. Mantyh PW, Clohisy DR, Koltzenburg M, Hunt SP (2002) Molecular mechanisms of cancer pain. Nat Rev Cancer 2:201-209. https://doi.org/10.1038/nrc747

47. Bian X, Dai H, Feng J, et al (2018) Prognostic values of abdominal body compositions on survival in advanced pancreatic cancer. Med (United States) 97https://doi.org/10.1097/MD.0000000000 010988

48. Freeman MD, Woodham MA, Woodham AW (2010) The role of the lumbar multifidus in chronic low back pain: a review. PM R 2:142-6; quiz $1 \mathrm{p}$ following 167. https://doi.org/10.1016/j.pmrj. 2009.11.006

49. Rice D, Nijs J, Kosek E et al (2019) Exercise-induced hypoalgesia in pain-free and chronic pain populations: state of the art and future directions. J Pain 20:1249-1266. https://doi.org/10.1016/j. jpain.2019.03.005
50. Sluka KA, Frey-Law L, Hoeger Bement M (2018) Exerciseinduced pain and analgesia? Underlying mechanisms and clinical translation Pain 159(Suppl):S91-S97. https://doi.org/10.1097/j. pain.0000000000001235

51. Santa Mina D, Brahmbhatt P, Lopez C et al (2017) The case for prehabilitation prior to breast cancer treatment. PM R 9:S305S316. https://doi.org/10.1016/j.pmrj.2017.08.402

Publisher's note Springer Nature remains neutral with regard to jurisdictional claims in published maps and institutional affiliations. 